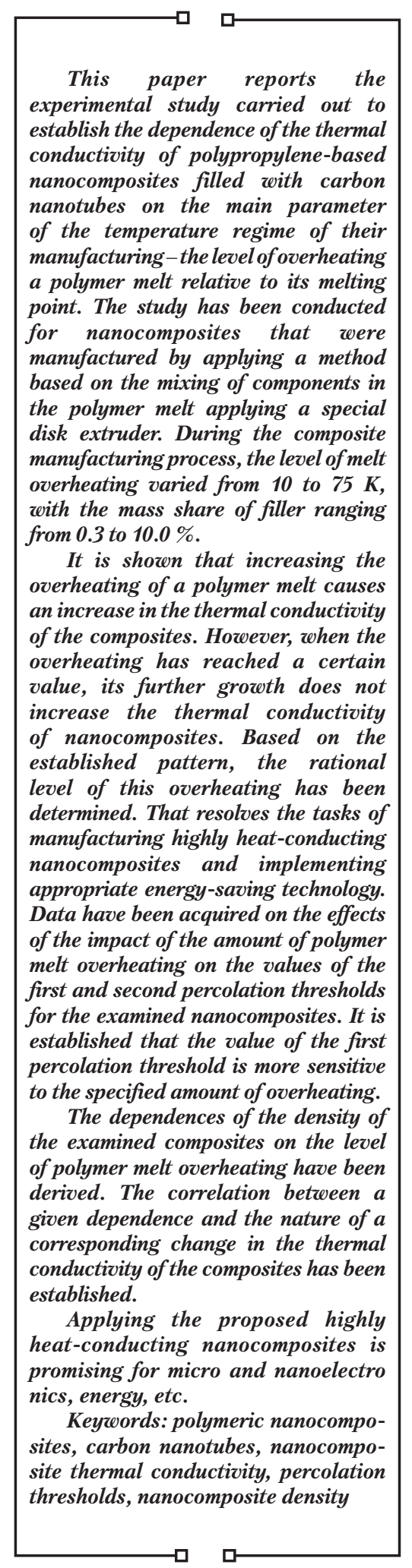

UDC 538.9:536.6

DOI: $10.15587 / 1729-4061.2021 .236915$

\title{
ESTABLISHING PATTERNS IN THE EFFECT OF TEMPERATURE REGIME WHEN MANUFACTURING NANOCOMPOSITES ON THEIR HEAT-CONDUCTING PROPERTIES
}

\section{Nataliia Fialko}

Corresponding author

Doctor of Technical Sciences, Professor, Corresponding Member of the National Academy of Sciences of Ukraine*

*Department of Thermophysics of Energy-Efficient Heat Technologies Institute of Engineering Thermophysics of National Academy of Sciences of Ukraine Mariyi Kapnist (Zheliabova) str., 2a, Kyiv, Ukraine, 03057 E-mail: nmfialko@ukr.net

Roman Dinzhos

Doctor of Technical Sciences, Professor Department of Physics and Mathematics V. O. Sukhomlynskyi National University of Mykolaiv Nikolska str., 24, Mykolaiv, Ukraine, 54006 Juli i Sherenkovski $\mathrm{PhD}$, Senior Researcher*

Nataliia Meranova $\mathrm{PhD}$, Senior Researcher*

Rais a $\mathbf{N a v r o d s k a}$ $\mathrm{PhD}$, Senior Scientific Researche, Leading Researcher*

Diana Izvorska

PhD, Associate Professor Department of Language and Specialized Education

Technical University of Gabrovo Hadzhi Dimitar str., 4, Gabrovo, Bulagaria, 5300

Volod y m y K orzhyk

Doctor of Technical Sciences, Head of Department Department of Electrothermal Processing Material E. O. Paton Electric Welding Institute of the National Academy of Sciences of Ukraine Bozhenka str., 11, Kyiv, Ukraine, 03680

Maxim Lazarenko Doctor of Physical and Mathematical Sciences

Department of Molecular Physics

Taras Shevchenko National University of Kyiv Volodymyrska str., 60, Kyiv, Ukraine, 01033

Neli Koseva $\mathrm{PhD}$, Professor

Laboratory of Phosphorus-Containing Monomers and Polymers Institute of Polymers at the Bulgarian Academy of Sciences Academician Georgi Bonchev str., block 103, Sofia, Bulgaria, 1113

\section{Introduction}

Replacing conventional metals and alloys with polymeric micro and nanocomposites is one of the current trends in technology. Important uses of polymeric composites include the application of their highly heat-conducting modifications. The latter can be widely used to fabricate such typical parts of elements of energy installations as heat exchange 
surfaces, in micro-and nanoelectronics - to divert heat from thermally-loaded elements of equipment, etc.

The thermal conductive properties of polymeric composites are known to depend significantly on the methods of their manufacturing.

When using a certain method, the specified properties are largely determined by the conditions of its implementation, in particular, the mode parameters of the appropriate equipment. As regards the class of methods for manufacturing polymeric composites, based on the mixing of components in the polymer melt, one of the important parameters that significantly influence the thermal conductive properties of polymeric composites is the level of melt overheating compared to the melting rate of the polymer. That makes it relevant to investigate the dependence of the thermal-physical properties of polymeric composite materials on a given parameter. At the same time, the task of finding a rational level of this overheating, which corresponds to highly heat-conducting polymeric composites is important, as well as the implementation of the appropriate energy-saving technology.

\section{Literature review and problem statement}

Many studies aimed to explore the thermal-physical properties of polymeric micro-nanocomposites [1-5]. Thus, [1] considers the possibilities of improving the thermal conductive properties of epoxy resins by applying nanofibers filled with different types of carbon nanostructures as a reinforcement material. Specifically, carbon nanotubes (CNTs), graphite plates, graphene nanoplates, and carbon soot were used as fillers. Work [2] addresses the thermal conductive characteristics of the "epoxy resin/multi-layered carbon nanotubes" composite during the modification of the latter in order to reduce the electrically conductive properties of the resulting material. Paper [3] gives an overview of the latest developments in the study of conductive polymeric composites. It provides an in-depth analysis of the data concerning the composites of conductive polymers with carbon materials, metal oxides, transient metals, etc. Study [4] describes experimental advances and theoretical progress in the field of heat transfer in polymers and their nanocomposites. Work [5] reports a theoretical study of the thermal properties of the "carbon nanotubes/epoxy resin" composites based on atomic modeling. In particular, it is shown that the effective thermal conductivity of the examined composites increases with an increase in the length of nanotubes and their volume share, with an increase in the degree of hardening of the epoxy resin and the temperature of the system.

However, there are almost no studies in the above papers [1-5] on the effects exerted by the manufacturing methods of polymeric composites on their thermal-physical performance. The results from studying these characteristics in the context of their dependence on manufacturing methods are reported in several works [6-10]. Paper [6] describes a comparative analysis of the thermal conductive properties of polymeric micro and nanocomposites based on polyethylene, obtained using methods that involve the mixing of components in a dry form or melt of the polymer matrix. The concentration dependences of thermal conductivity coefficients for polyethylene filled with CNTs or aluminum microparticles are given. It has been shown that the use of a method based on the mixing of components in the polymer melt could provide micro and nanocomposites with significantly higher thermal conductive properties. At the same time, for polyethylene filled with CNTs, this effect is more significant than in the case of using aluminum microparticles as a filler. The specificity of manufacturing the foam from polyvinyl formaldehyde, filled with multi-layer carbon nanotubes, is considered in [7]. The values for the thermal conductivity coefficients of these materials at different values of their temperature were determined. It is noted that a given method for manufacturing polymeric composite materials is simple and scalable. Study [8] addresses the evaluation of the thermal-physical properties of epoxy nanocomposites manufactured using a method of in situ polymerization and reinforced with nanodiamonds, as well as the epitaxial nitride of boron. In [9], the method of mixing in solutions with further processing by compounding in a melt is used as a method for preparing nanocomposites. The cited study focuses on the thermal and tribological properties of "polymer/graphene" composites. In paper [10], an electrospinning method is used in the manufacture of flexible highly heat-conducting nanocomposites in order to connect the sheets made of boron nitride with the oriented CNTs. It is noted that a significant improvement in the thermal conductivity of composites is due to the reduction of interphase thermal resistance between neighboring nanotubes.

The above studies [6-10] consider the thermal conductive properties of composites manufactured by using known methods. When applying new methods, the task arises at the stage of their scientific and technical justification to establish the dependence of these properties on the regime parameters of devices for implementing those methods.

Thus, the available body of research into the thermal-physical properties of polymeric micro and nanocomposites does not cover new methods for obtaining them. In particular, it is important to establish the patterns of influence of the regime parameters of new technologies for manufacturing composites on their thermal-physical properties and to choose rational values for these parameters.

It also follows from our review that when obtaining highly heat-conducting modifications of polymeric composites, special attention is paid to the use of various carbon nanostructures as fillers, and primarily carbon nanotubes, as fillers.

\section{The aim and objectives of the study}

The aim of this work is to establish patterns of influence exerted on the thermal conductive properties of polymeric nanocomposites by the temperature mode of their manufacturing when using a method based on the mixing of components in the polymer melt involving a special disk extruder. This would make it possible to choose a rational regime that corresponds to the manufacturing of nanocomposites whose thermal conductivity is close to maximal at the predefined concentration of a filler.

To achieve the set aim, the following tasks have been solved:

- to perform a set of experimental studies to establish the dependence of the thermal conductivity of the resulting composites on the level of overheating the polymer melt $\Delta T$ relative its melting temperature and to determine, based on this, the rational values for the specified overheating;

- to identify patterns of the effect of the overheating magnitude $\Delta T$ on the density of polymeric composites over a wide temperature range. 


\section{The study materials and methods}

In the current work, in order to obtain polymeric nanocomposites, we used a method based on the mixing of components in the polymer melt by applying a special disk extruder [11]. The polymers' physical properties were determined using standard and improved procedures.

This work aims to acquire relevant information about highly heat-conducting polymeric nanocomposites based on polypropylene, filled with carbon nanotubes, while the mass fraction of filler varies in a wide range.

\section{1. A method for manufacturing polymeric nanocom-} posites

The structural diagram of the extruder involved in the implementation of the proposed method for manufacturing nanocomposites is shown in Fig. 1. The composite powder mixture 3 is placed in mold 1 , which is heated by electric heater 2 to a temperature higher than the melting point of the polymer by the magnitude $\Delta T$. The components of the composite are mixed using a rotating metal piston 4. The latter is gradually immersed in the polymer melt during the mixing process. At the end of the mixing process, the composite is removed from the mold through hole 5 .

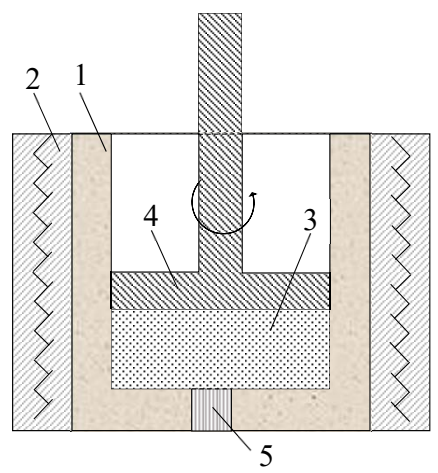

Fig. 1. The structural diagram of the extruder: 1 - mold; 2 - electric heater; 3 - powder composite; 4 - rotating piston; 5 - hole at the bottom of the mold

The process of obtaining polymeric nanocomposites is completed using a hot-pressing method in a special installation. In it, the composite is heated to a temperature exceeding $20{ }^{\circ} \mathrm{C}$ and is aged at this temperature for 15 to 20 minutes. Then the resulting samples are pressed; they are given the desired shape.

The characteristics of carbon nanotubes used as fillers are described in [6].

4. 2. Methods to study the properties of polymeric nanocomposites

During our research, the thermal conductivity coefficient and the density of polymeric nanocomposites were to be experimentally determined. The thermal conductivity coefficient of polymeric composites was determined using the modified IT- $\lambda$ - 400 device. A hydrostatic weighing method was applied to find the density of the examined materials.

The experimental study into the properties of the examined polymeric nanocomposites was carried out when the mass share of carbon nanotubes changed from 0.3 to $10 \%$. At the same time, in the process of obtaining the composites, the level of overheating the polymer melt $\Delta T$ relative to its melting temperature varied from 10 to $75^{\circ} \mathrm{C}$.

\section{The results of studying the properties of polymeric nanocomposites filled with carbon nanotubes}

5.1. The study results on determining the thermal conductivity coefficient of polymeric nanocomposites

Fig. 2 shows the results of our experimental study of the thermal conductivity coefficients of the examined composites depending on the level of overheating $\Delta T$ at different values of the mass share of CNTs. The experimental points in the figure indicate the margin of error in determining their values.

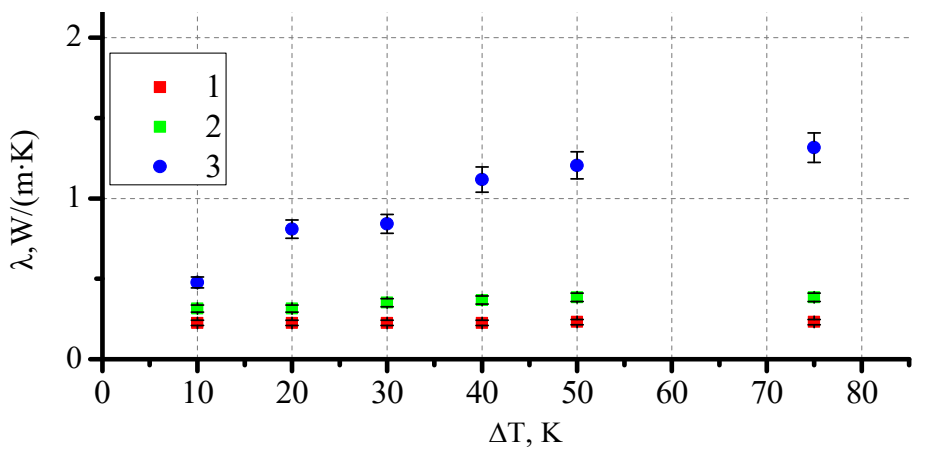

$a$

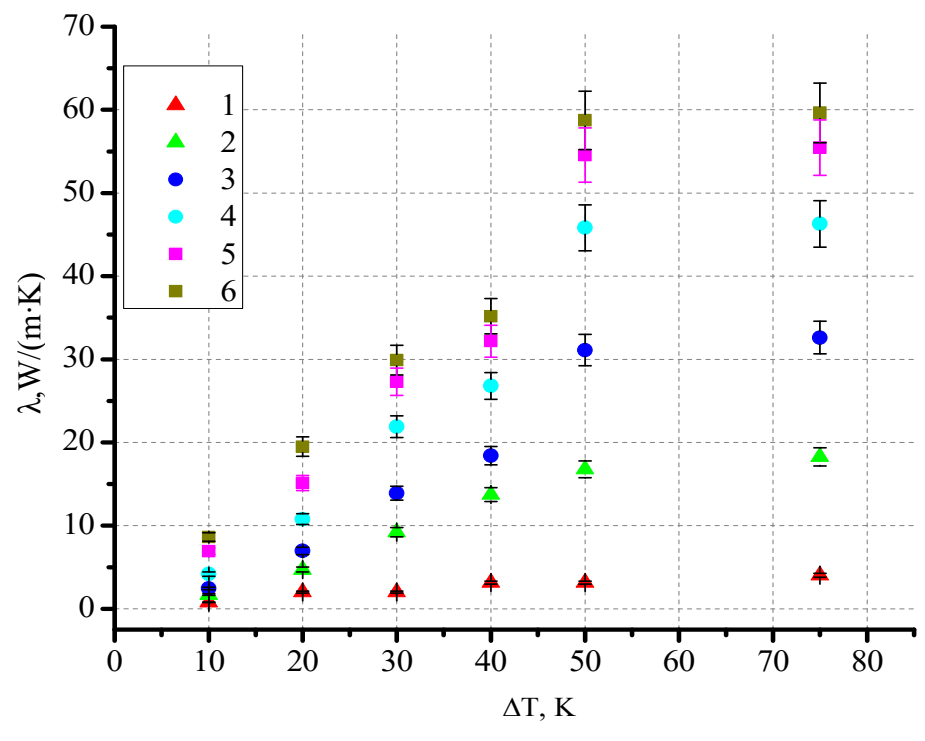

$b$

Fig. 2. Dependence of the thermal conductivity coefficient $\lambda$ of the polypropylene-based nanocomposites filled with carbon nanotubes on the overheating of polymer melt $\Delta T$ at various values of the mass share of filler $\omega: a-1-\omega=0.3 \% ; 2-1 \% ; 3-1.3 \%$;

$$
\begin{gathered}
b-4-\omega=2.0 \% ; 5-2.5 \% ; 6-3.0 \% ; \\
7-5.0 \% ; 8-7.0 \% ; 9-10.0 \%
\end{gathered}
$$

Fig. 3 illustrates the values of the first and second threshold of percolation at different values of the overheating $\Delta T$. 


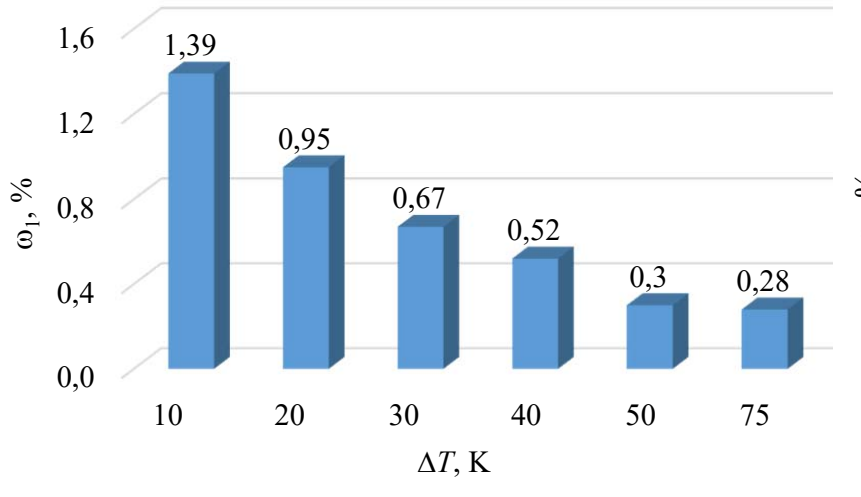

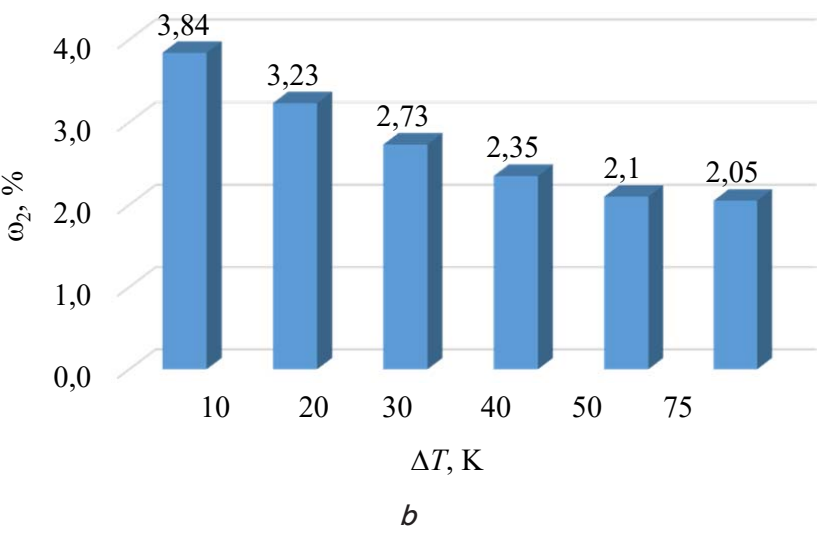

Fig. 3. Values of the mass share of filler $\omega$ in the composite based on polypropylene, filled with CNTs, at different levels of the overheating of a polymer melt $\Delta T: a$-values of the mass share of filler $\omega_{1}$ that correspond to the first threshold of percolation; $b$-values of the mass share of filler $\omega_{2}$ that correspond to the second threshold of percolation

The percolation thresholds shown in Fig. 3 correspond to the values of the mass share of the filler at which there are sharp changes in the coefficient of the thermal conductivity of the composite.

\section{2. The study results on determining the density of} polymeric nanocomposites

The density of the examined polymeric nanocomposites was investigated in a temperature interval of $300-450 \mathrm{~K}$.

Fig. 4 shows the results of our experimental study to determine a change in the density of the composites depending on the overheating of the polymer melt $\Delta T$ at the different levels of the temperature of these composites. The experimental points in the figure indicate the margin of error in determining their values.

The dependences $\rho=f(\Delta \mathrm{T})$ shown in Fig. 4 cover the temperature range from an ambient temperature to the temperature close to a polymer melting point.

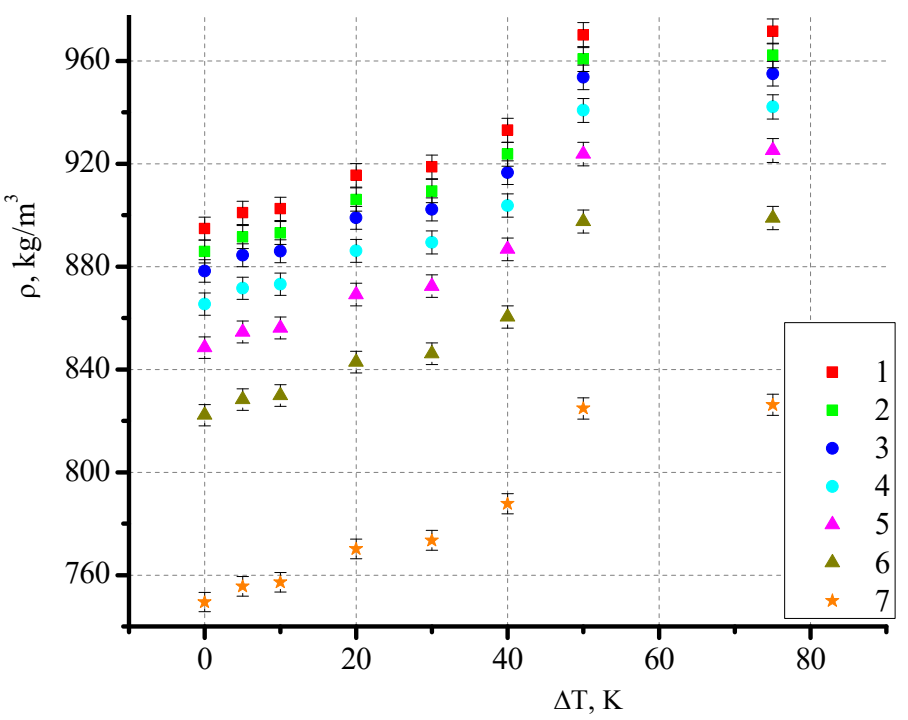

Fig. 4. Dependence of density $\rho$ of the nanocomposite based on polypropylene, filled with carbon nanotubes, on the polymer melt overheating $\Delta T$ at different composite temperatures: $1-T=300 \mathrm{~K}$; $2-325 \mathrm{~K} ; 3-350 \mathrm{~K} ; 4-375 \mathrm{~K} ; 5-400 \mathrm{~K} ; 6-425 \mathrm{~K} ; 7-450 \mathrm{~K}$

\section{Discussion of results of studying the physical properties of polymeric nanocomposites filled with carbon nanotubes}

6. 1. Discussion of the study results on determining the thermal conductivity coefficient of polymeric nanocomposites

Our experimental data indicate that the thermal conductivity coefficient $\lambda$ of the examined polymeric nanocomposites may generally depend significantly on the level of polymer melt overheating $\Delta T$ (Fig. 2). As one can see, for all the values of the mass share of filler $\omega$, the thermal conductivity coefficient increases with an increase in $\Delta T$ from 10 to $50 \mathrm{~K}$. At the same time, in a given interval, the $\Delta T$ changes in the dependence $\lambda=f(\Delta \mathrm{T})$ have certain features at different $\omega$ values. First, the larger $\omega$, the more significant the impact of the growth in $\Delta T$ on an increase in the composite's thermal conductivity coefficient. Thus, at $\omega=2.0 \%$, the thermal conductivity coefficient $\lambda$ varies from 0.8 to $3.15 \mathrm{~W} /(\mathrm{m} \cdot \mathrm{K})$ with an increase in $\Delta T$ from 10 to $50 \mathrm{~K}$. For $\omega=10.0 \%$, the corresponding changes are 8.62 and $58.72 \mathrm{~W} /(\mathrm{m} \cdot \mathrm{K})$. In addition, the nature of the dependence $\lambda=f(\Delta \mathrm{T})$ is slightly different at different $\omega$ values. Specifically, at $0.3 \% \leq \omega \leq 2.5 \%$, this dependence is close to linear. At $3.0 \% \leq \omega \leq 10.0 \%$, there is an uneven rate of increase in $\lambda$ with an increase in the level of overheating $\Delta T$. Note a very sharp increase in the thermal conductivity of the composite with an increase in $\Delta T$ from 40 to $50 \mathrm{~K}$. And this increase is even more significant the greater the mass share of filler. As for the increase in the level of composite overheating $\Delta T$ from $50 \mathrm{~K}$ to $75 \mathrm{~K}$, this increase in a given interval does not lead to a noticeable change in the composite $\lambda$.

The described pattern of the effect of overheating $\Delta T$ on the thermal conductivity of the composites studied is associated with the following events. When $\Delta T$ is increased due to the decrease in the viscosity of the polymer melt, CNTs are distributed more evenly in it. That leads to an increase in the efficiency of the formation of percolation structures from CNTs, and, as a result, to an increase in the thermal conductivity of nanocomposites. When $\Delta T$ is increased to a certain level (in a given case, to $50 \mathrm{~K}$ ), the even distribution of the filler in the polymer melt is achieved, close to the 
limit under these conditions, and, accordingly, the values $\lambda$ of the composites close to maximally possible.

Improving the efficiency of the formation of percolation structures with growth in $\Delta T$ is associated with a decrease in the values of both the first and second thresholds of percolation (Fig. 3). At the same time, in the interval of $\Delta T$ change under consideration, the reduction of the first threshold of percolation (about 5 times), when compared to the second threshold (less than 2 times), is more significant.

These results of our research make it possible to select the rational level of the polymer overheating $\Delta T$, which resolves the task of manufacturing highly heat-conducting nanocomposites.

In a general case, the choice of this rational $\Delta T$ level is determined by the following considerations:

- the overheating $\Delta T$ is limited on the top to the temperature of the polymer's destruction;

- the amount of overheating corresponds to manufacturing a nanocomposite whose thermal conductivity is close to maximal at the predefined concentration of filler;

- for the implementation of energy-saving technology, the overheating level $\Delta T$ is limited to the region of a significant change in $\lambda$ with the growth in $\Delta T$.

Taking these considerations into account, the rational value of the overheating level $\Delta T$ for the examined nanocomposites is $50 \mathrm{~K}$.

\section{2. Discussion of the study results on determining} the density of polymeric composites

According to the data acquired, the pattern of change in the density of composites $\rho$, depending on the level of the polymer melt overheating $\Delta T$, is correlated with the corresponding dependence for their thermal conductivity coefficient (Fig. 2, 4). Specifically, the density $\rho$ increases with an increase in the overheating $\Delta T$ to $50 \mathrm{~K}$ over the entire range of temperature change in the composite ( $300 \mathrm{~K}<T<450 \mathrm{~K}$ ). With further growth of the level of overheating from $50 \mathrm{~K}$ to $75 \mathrm{~K}$, a change in the density of composites $\rho$ is negligible.

It is noteworthy that the dependence of the density of composites $\rho$ on the level of the polymer melt overheating $\Delta T$ is less significant than a given dependence for the thermal conductivity factor. For example, at $\omega=3.0 \%$, with an increase in $\Delta T$ from 10 to $50 \mathrm{~K}$, the thermal conductivity coefficient of the composite in question increases by 12.75 times, and the density of the material at $450 \mathrm{~K}$ is only by 1.08 times.

The increase in the density of nanocomposites with an increase in the level of the polymer overheating $\Delta T$ is associated with the above-described effect of increasing the degree of uniformity of the distribution of CNTs in the polypropylene matrix when the temperature of its melt increases. A given increase generates a large branching of the percolation structures made from CNTs. This, in turn, leads to increased electromagnetic interaction between CNTs and polypropylene. The latter determines the increase in the density of the composite material.

Fig. 4 shows that the density of polymeric composite materials falls with the increase in their temperature at all values of the level of the polymer overheating $\Delta T$. At the same time, there is a tendency to increase the intensity of this fall with the increase in the temperature of the composite. Thus, at $\Delta T=20 \mathrm{~K}$, with the temperature of the polymeric composite material rising from 300 to $325 \mathrm{~K}$, the resulting drop is
$9.36 \mathrm{~kg} / \mathrm{m}^{3}$, and with an increase in temperature from 425 to $450 \mathrm{~K}-72.67 \mathrm{~kg} / \mathrm{m}^{3}$.

The work reported here concerns only the thermal conductivity and density of polymeric nanocomposites of a certain type. It describes a study into the dependence of these properties on only one regime parameter of their manufacturing - the level of polymer overheating relative to its melting point.

Further research into the physical properties of polymeric nanocomposites may involve studying their characteristics such as specific heat capacity, heat crystallization, etc. At the same time, it is interesting to continue research aimed at establishing the dependence of the properties of composites on the various regime parameters related to manufacturing them - the time of mixing components, the speed of rotation of the extruder, etc.

\section{Conclusions}

1. We have performed a set of experimental studies to determine the dependence of the thermal conductivity coefficients of the nanocomposites based on polypropylene, filled with CNTs, on the basic parameter for the temperature regime of manufacturing them - the value of the polymer melt overheating relative to its melting temperature. The composites studied were fabricated using a method based on the mixing of components in the polymer melt applying a special disk extruder. The experiment was conducted in a wide range of change in the level of polymer melt overheating (from 10 to $75 \mathrm{~K}$ ) at the different values of the mass share of CNTs (from 0.3 to $10.0 \%$ ). It has been shown that an increase in the level of the polymer overheating can lead to a significant (by about 7 times) increase in the thermal conductivity of composites. It has been established that the effect of the level of the polymer melt overheating is even more significant the higher the mass share of filler. The interpretation of the derived dependence of the thermal conductivity of composites on the level of overheating of the polymer melt from the position of formation of percolation structures is given. We have established that the thermal conductivity of the composites has changed slightly in the range of change in the polymer melt overheating from 50 to $75 \mathrm{~K}$ at all studied values of the mass share of the filler. The rational level of polymer melt overheating has been determined, which resolves the task of manufacturing highly heat-conducting nanocomposites and implementing the appropriate energy-saving technology.

2. We have derived the experimental dependences of the density of the polymeric composites in question on the level of polymer melt overheating relative to its melting temperature for a wide range of change in the temperature of composites $(300 \leq \mathrm{T} \leq 450 \mathrm{~K})$. It is shown that the nature of change in the density of composites depending on the magnitude of the specified overheating is correlated with the corresponding dependence for the coefficient of thermal conductivity. The patterns of the effect of the value of the polymer melt overheating on the density of the resulting nanocomposites based on changes in the degree of branching of the emerging percolation structures of the filler and its electromagnetic interaction with the polymer matrix have been explained. 


\section{Acknowledgments}

This study was partially funded by the DR project No. 012 U110115 (Order No. 257 as of 25.02.2021) for the fundamental and applied research and scientific and technological advancements at the institutions of higher education and scientific establishments for 2021, which are managed by the Ministry of Education and Science of Ukraine.

\section{References}

1. Datsyuk, V., Trotsenko, S., Trakakis, G., Boden, A., Vyzas-Asimakopoulos, K., Parthenios, J. et. al. (2020). Thermal properties enhancement of epoxy resins by incorporating polybenzimidazole nanofibers filled with graphene and carbon nanotubes as reinforcing material. Polymer Testing, 82, 106317. doi: https://doi.org/10.1016/j.polymertesting.2019.106317

2. Wang, R., Xie, C., Luo, S., Xu, H., Gou, B., Zeng, L. (2020). Preparation and properties of MWCNTs-BNNSs/epoxy composites with high thermal conductivity and low dielectric loss. Materials Today Communications, 24, 100985. doi: https://doi.org/10.1016/ j.mtcomm.2020.100985

3. Namsheer, K., Rout, C. S. (2021). Conducting polymers: a comprehensive review on recent advances in synthesis, properties and applications. RSC Advances, 11 (10), 5659-5697. doi: https://doi.org/10.1039/d0ra07800j

4. Xu, X., Chen, J., Zhou, J., Li, B. (2018). Thermal Conductivity of Polymers and Their Nanocomposites. Advanced Materials, 30 (17), 1705544. doi: https://doi.org/10.1002/adma.201705544

5. Mohammad Nejad, S., Srivastava, R., Bellussi, F. M., Chávez Thielemann, H., Asinari, P., Fasano, M. (2021). Nanoscale thermal properties of carbon nanotubes/epoxy composites by atomistic simulations. International Journal of Thermal Sciences, 159, 106588. doi: https://doi.org/10.1016/j.ijthermalsci.2020.106588

6. Fialko, N. M., Dinzhos, R. V., Sherenkovskiy, Y. V., Meranova, N. O., Navrodskaya, R. A. (2017). Heat conductivity of polymeric micro- and nanocomposites based on polyethylene at various methods of their preparation. Industrial Heat Engineering, 39 (4), 21-26. doi: https://doi.org/10.31472/ihe.4.2017.03

7. Anis, B., Fllah, H. E., Ismail, T., Fathallah, W. M., Khalil, A. S. G., Hemeda, O. M., Badr, Y. A. (2020). Preparation, characterization, and thermal conductivity of polyvinyl-formaldehyde/MWCNTs foam: A low cost heat sink substrate. Journal of Materials Research and Technology, 9 (3), 2934-2945. doi: https://doi.org/10.1016/j.jmrt.2020.01.044

8. Zhang, Y., Choi, J. R., Park, S.-J. (2017). Thermal conductivity and thermo-physical properties of nanodiamond-attached exfoliated hexagonal boron nitride/epoxy nanocomposites for microelectronics. Composites Part A: Applied Science and Manufacturing, 101, 227-236. doi: https://doi.org/10.1016/j.compositesa.2017.06.019

9. Uyor, U., Popoola, A., Popoola, O., Aigbodion, V. (2019). Effects of titania on tribological and thermal properties of polymer/graphene nanocomposites. Journal of Thermoplastic Composite Materials, 33 (8), 1030-1047. doi: https://doi.org/ $10.1177 / 0892705718817337$

10. Yang, L., Zhang, L., Li, C. (2020). Bridging boron nitride nanosheets with oriented carbon nanotubes by electrospinning for the fabrication of thermal conductivity enhanced flexible nanocomposites. Composites Science and Technology, 200, 108429. doi: https://doi.org/10.1016/j.compscitech.2020.108429

11. Dinzhos, R., Fialko, N., Prokopov, V., Sherenkovskiy, Y., Meranova, N., Koseva, N. et. al. (2020). Identifying the influence of the polymer matrix type on the structure formation of microcomposites when they are filled with copper particles. Eastern-European Journal of Enterprise Technologies, 5 (6 (107)), 49-57. doi: https://doi.org/10.15587/1729-4061.2020.214810 